\title{
Lob für die Zahnärzteschaft aus der Politik
}

Es war der Abend, an dem gleich mehrere Philosophen für die Grußworte aus der Politik bemüht wurden. Doch zum traditionellen Neujahrsempfang von Bundeszahnärztekammer (BZÄK) und Kassenzahnärztlicher Bundesvereinigung (KZBV) waren sich die Gäste aus der deutschen Gesundheitspolitik vor allem in einem einig: Die Zahnärzteschaft hat in Zeiten der Flüchtlingskrise mit ihrem Engagement eine überzeugende Performance abgeliefert. Lob dafür gab es von allen Seiten. BZ̈̈KPräsident Peter Engel wies jedoch darauf hin, dass uneindeutige Regelungen Flüchtlinge, Behandler und Ämter gleichermaßen verunsicherten und forderte die zuständigen Stellen auf, klare Regelungen zu treffen.

Neben allem Lob blieb in den politischen Grußworten nicht unerwähnt, dass nach den vielen gesundheitspolitischen Reformen 2015 auch in diesem Jahr noch Reformprojekte anstünden, von denen auch die Zahnärzte betroffen seien. Darin waren sich die gesundheitspolitischen Sprecher der Bundestagsfraktionen, Maria Michalk (CDU/CSU), Birgit Wöllert (Linke) und Harald Terpe (Bündnis 90/Grüne), sowie der Vorsitzende des Bundestagsausschusses für Gesundheit, Edgar Franke (SPD), ebenfalls einig. In Fragen der Prävention sei die Zahnärzteschaft jedoch weit voran und über die von der Europäischen Union (EU) installierten Richtlinien hinausgegangen, sagte Gesundheitspolitiker Terpe. Im Hinblick auf die Pflegereform und die aufsuchende Zahnmedizin betonte Michalk: „Es sind viele kleine Dinge, die großes Bewirken können.“ Auch Franke hob die Versorgung von Pflegebedürftigen hervor.

Mit der EU schnitt Terpe ein Thema an, das die Zahnärzteschaft aktuell umtreibt: Kommt über die Berufsanerkennungsgepflogenheiten der Bachelor-Zahnarzt auf Deutschland zu? "Ich habe noch niemanden gehört, der das möchte“, sagte der Gesundheitspolitiker. Er sprach sich für eine neue Approbationsordnung und weniger Modellstudiengänge in Deutschland aus, damit „nicht mit den Modellstudiengängen eine neue Realität geschaffen“"werde.
In der Zahnärzteschaft rannte er damit offene Türen ein. Laut BZÄK-Präsident Engel gibt es die Befürchtung einer „Einführung des Bachelors durch die Hintertür“. Er sprach sich für eine neue, moderne Approbationsordnung aus. „Modellstudiengänge können punktuell sinnvoll sein, bleiben aber immer eine Notlösung“, machte Engel deutlich. Der Vorstandsvorsitzende der KZBV, Wolfgang Eßer, schlug einen großen politischen Bogen. Vor den aktuellen politischen Problemlagen seien die „zahnärztlichen Themen vergleichsweise klein“, allerdings setze er trotzdem auf kluge politische Entscheidungen: „Gerade vor dem Hintergrund großer demografischer Herausforderungen, die auf uns zukommen, müssen die notwendigen Weichen richtig gestellt werden. " Um die zahnärztliche Versorgung auch in $\mathrm{Zu}$ kunft aufrechtzuerhalten, forderte er die Politik auf, die Regelungen zu arztgruppengleichen Medizinischen Versorgungszentren (MVZ) wieder zurückzunehmen: „MVZ sind nicht versorgungsfördernd, weil sie nur ertragsorientiert arbeiten.“

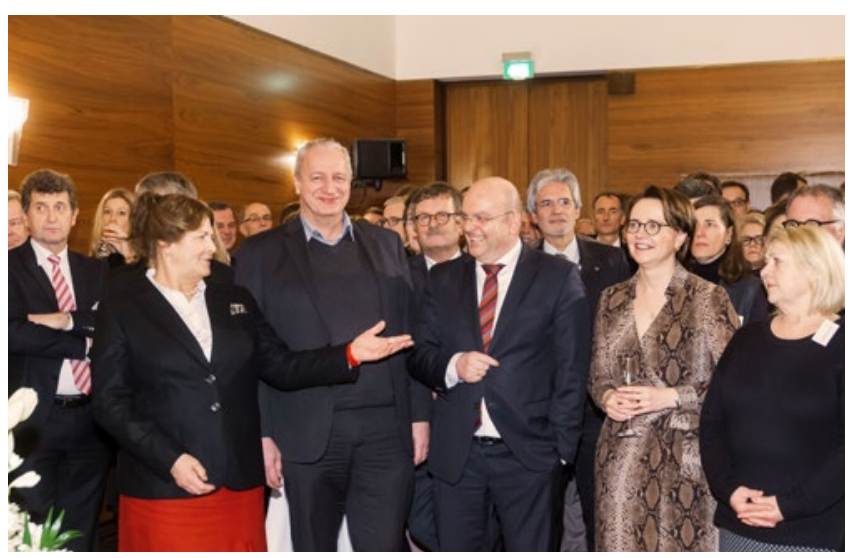

Politiker aller Bundestagsfraktionen lobten beim Neujahrsempfang von BZÄK und KZBV die Zahnärzteschaft für ihren Einsatz.

\section{Deutliches Wachstum}

\section{Zahl der Beschäftigten im Gesundheitswesen steigt}

Um satte zehn Prozent ist die Beschäftigung im Gesundheitswesen in den vergangenen fünf Jahren gestiegen. Damit ist klar: Das Gesundheitswesen wird als Arbeitgeber immer wichtiger und beliebter. Ende 2014 waren 5,2 Millionen Menschen im medizinischen Bereich beschäftigt - über 100.000 (zwei Prozent) mehr als noch im Jahr zuvor. Dies teilte das Statistische Bundesamt mit. Den größten Stellenzuwachs verzeichnete die Altenpflege mit einem Plus von vier Prozent (plus 21.000 Beschäftigte). Bei Zahnärzten und Humanmedizinern (mit ihren Angestellten) waren es etwa 2,1 Prozent mehr Beschäftigte als im Vorjahr (plus 9.000 Beschäftigte). Im Vergleich zwischen den beiden Arztgruppen verzeichnen die Humanmediziner den deutlich größeren Zuwachs (plus 8.000 Beschäftigte). In den
Zahnarztpraxen waren 2014 insgesamt 72.000 Zahnärzte und 191.000 zahnmedizinische Fachangestellte beschäftigt, die Zahlen in zahntechnischen Berufen blieben bei 55.000 in den vergangenen Jahren relativ konstant. Und noch eines stellten die Statistiker fest: Der Anteil der Frauen im Gesundheitswesen wächst in allen Berei-

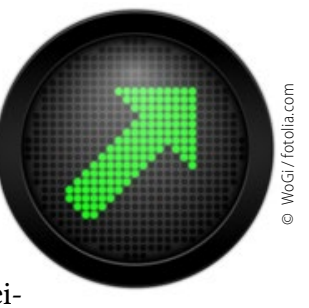
chen. Von allen Beschäftigten im Gesundheitswesen war nach Angaben des Statistischen Bundesamts gut die Hälfte teilzeit- oder geringfügig beschäftigt. Den Zahlen zufolge sind in der Gesundheitswirtschaft zwischen 2004 und 2014 insgesamt mehr als 900.000 neue Arbeitsplätze entstanden. Dies entspricht einem Zuwachs von mehr als 20 Prozent. 